University of Wollongong

Research Online

Faculty of Social Sciences - Papers (Archive) Faculty of Arts, Social Sciences \& Humanities

2014

Generating procedural and conceptual knowledge of fractions by preservice teachers

Mohan Chinnappan

University of South Australia, Mohan.Chinnappan@unisa.edu.au

Patricia Forrester

University of Wollongong, tricia@uow.edu.au

Follow this and additional works at: https://ro.uow.edu.au/sspapers

Part of the Education Commons, and the Social and Behavioral Sciences Commons

Research Online is the open access institutional repository for the University of Wollongong. For further information contact the UOW Library: research-pubs@uow.edu.au 


\title{
Generating procedural and conceptual knowledge of fractions by pre-service teachers
}

\begin{abstract}
Knowledge that teachers bring to the teaching context is of interest to key stakeholders in improving levels of numeracy attained by learners. In this regard, the centrality of, and the need to investigate, the quality of teachers' mathematical knowledge for teaching mathematics has been gaining momentum in recent years. There is a general consensus that teachers need a robust body of content and pedagogical knowledge related to mathematics and that one impacts on the other. However, in current debates about this interconnection between content knowledge and pedagogical content knowledge, there is limited analysis about the procedural-conceptual nature of content knowledge that, we argue, has significant impact on the development of pedagogical content knowledge. In this report, this issue is investigated by examining the state of procedural and conceptual knowledge of two cohorts of pre-service teachers and analyzing the impact of a representational reasoning teaching and learning (RRTL) approach aimed at supporting a balanced development of these two dimensions of Content Knowledge.
\end{abstract}

\section{Keywords}

knowledge, teachers, conceptual, service, procedural, generating, pre, fractions

\section{Disciplines}

Education | Social and Behavioral Sciences

\section{Publication Details}

Chinnappan, M. \& Forrester, T. (2014). Generating procedural and conceptual knowledge of fractions by pre-service teachers. Mathematics Education Research Journal, 26 (4), 871-896. 


\title{
Generating procedural and conceptual knowledge of fractions by pre-service teachers
}

\author{
Mohan Chinnappan • Tricia Forrester
}

Received: 3 March 2013 / Revised: 2 June 2014 / Accepted: 27 June 2014 /

Published online: 15 July 2014

(C) Mathematics Education Research Group of Australasia, Inc. 2014

\begin{abstract}
Knowledge that teachers bring to the teaching context is of interest to key stakeholders in improving levels of numeracy attained by learners. In this regard, the centrality of, and the need to investigate, the quality of teachers' mathematical knowledge for teaching mathematics has been gaining momentum in recent years. There is a general consensus that teachers need a robust body of content and pedagogical knowledge related to mathematics and that one impacts on the other. However, in current debates about this interconnection between content knowledge and pedagogical content knowledge, there is limited analysis about the procedural-conceptual nature of content knowledge that, we argue, has significant impact on the development of pedagogical content knowledge. In this report, this issue is investigated by examining the state of procedural and conceptual knowledge of two cohorts of pre-service teachers and analyzing the impact of a representational reasoning teaching and learning (RRTL) approach aimed at supporting a balanced development of these two dimensions of Content Knowledge.
\end{abstract}

Keywords Pre-service teacher knowledge · Fractions · Procedural knowledge · Conceptual knowledge $\cdot$ Mathematical knowledge for teaching

\section{Background}

Discussions about mathematics teaching and teacher quality bring into focus the knowledge base that drives teachers' practice. More than two decades ago, Shulman (1986), in examining the kinds of knowledge that are important to teachers' work, identified two categories of knowledge that were necessary for effective practice, namely: subject matter (content) knowledge and pedagogical content knowledge.

M. Chinnappan $(\bowtie)$

School of Education, University of South Australia, GPO Box 2471, Adelaide, SA 5001, Australia e-mail: Mohan.Chinnappan@unisa.edu.au

T. Forrester

University of Wollongong, Wollongong, Australia

e-mail: tricia@uow.edu.au 
Within mathematics education, there is an emerging consensus that teachers' professional knowledge for teaching mathematics must be rooted in a robust body of Content Knowledge and Pedagogical Content Knowledge (Frid et al. 2009; Walshaw 2012). Research indicates that teachers' mathematics content knowledge contributes significantly to their students' achievement (Bobis et al. 2012; Senk et al. 2012).

The seminal work of Shulman has spawned a number of studies in various subject domains including mathematics. Ball and her associates (Ball et al. 2005, 2008; Hill et al. 2008), in seeking to analyze the knowledge necessary for teaching mathematics effectively, have developed two major strands for these knowledge clusters namely: content knowledge and pedagogical content knowledge. Content knowledge refers to knowledge of the concepts, principles, procedures, and conventions of mathematics, and pedagogical content knowledge involves teachers' understanding of students' mathematical thinking (including conceptions and misconceptions) and representing mathematics content knowledge in a learner-friendly manner. In unpacking the content knowledge category further, Ball et al. (2005), identified common content knowledge (knowledge of mathematics common to most educated adults) and specialized content knowledge (specific and detailed knowledge of mathematics required to teach it). It is the latter knowledge that is of interest to the present study but in the context of fractions.

While the connections between content knowledge and pedagogical content knowledge continue to receive attention (Ma 1999; Ball et al. 2005), discussions about the nature of content knowledge that lends itself to transformation to pedagogical content knowledge and vice versa have commanded limited visibility among researchers. For example, Chick (2010) analyzed the link between teachers' understanding of ratio and their knowledge for teaching ratio and suggested that mathematical considerations have important pedagogical consequences (p. 151). What are these "mathematical considerations"? We argue that conceptual and procedural underpinnings of the mathematics content that teachers bring to their teaching constitute important ingredients of the mathematical considerations which Chick (2010) alluded to.

Pre-service understanding of fractions and misconceptions

Within most primary mathematics curricula, fractions receive a great deal of attention. Conceptually, fractions present a hurdle as students attempt to transfer their understanding of whole numbers to a new but related class of numbers. Several researchers (Mack 2001; Lamon 2007) and, more recently, Harvey (2012) showed us that fractions are a problematic area of learning for both students and their teachers. Investigations conducted by Tirosh (2000) showed that pre-service teachers tended to have a mechanical understanding of fractions and this knowledge is less likely to help them support their students develop conceptual knowledge that girds fraction problems that involve division. A potential misconception includes ordering of fractions by focusing on the denominator or numerator. A key construct that underpins the learning of fractions is the part-whole relationship. Students and teachers need to understand the part-whole relationship between the numerator and the denominator or they may treat these numbers as wholes. This misconception could also lead students to compute by adding or subtracting the numerators and denominators separately. 
Ball (1990) found that prospective teachers were able to solve fraction division problems using the traditional invert-and-multiply algorithm but only a few could provide the correct pictorial representation. We regard Ball's work as representing a case of future teachers having developed procedural or algorithmic knowledge without understanding the conceptual base. Following an extensive review of research in preservice teachers' difficulties with fractions, Newton (2008) commented that knowledge that underpins the teaching of fractions is complex and multidimensional and that "more studies should examine knowledge from multiple perspectives, including an examination of correct solution methods,' (p.26) an approach that was adopted in the present study.

\section{Procedural and conceptual knowledge}

Broadly speaking, procedural knowledge involves understanding the rules and routines of mathematics while conceptual knowledge involves an understanding of mathematical relationships. While these two strands of knowledge are differentiable, they are related and impact on each other. Schneider and Stern (2010) have highlighted the complexities of the connections between procedural and conceptual knowledge, and the dependency of one on the other. They argue that the study of the development of these knowledge components continues to be a legitimate concern for mathematics teachers and researchers alike, suggesting that research into teaching and learning to examine their parallel development is warranted.

Within the context of teaching primary school fractions, Mack (2001) suggests that children's uses of strategies for representing and solving fraction problems involve the application of both these knowledge strands. In decoding and solving fraction problems, the relative roles and connections between these two dimensions of knowledge need further clarification. The debate on this issue appears to proceed along three lines. One view is that children learn conceptual knowledge of fractions before the development of procedural knowledge (Groth and Bergner 2006). A second view is that children learn procedural knowledge before conceptual knowledge (Baroody et al. 2005). Finally, it would seem that children's conceptual knowledge and procedural knowledge grow in tandem with one building on the other (Schneider and Stern 2010). This study is based on the latter assumption that conceptual knowledge plays a significant role in learners' generation and application of procedural knowledge. At the same time, as learners' procedural knowledge develops, it can influence their conceptual knowledge. Thus, the relation between the two strands of knowledge is not unidirectional as suggested by Rittle-Johnson and Alibali (1999). The argument about interdependency between the conceptual and procedural has gained support in a recent research by Hallett et al. (2010) suggesting that (a) some children rely on procedural knowledge to inform conceptual knowledge and (b) those who rely on conceptual knowledge of fractions tend to have an advantage over those who rely on procedural knowledge.

The above line of thinking and associated findings suggests that prospective and practicing teachers need to have a sound understanding of both knowledge categories that involve fractions. That is, despite a growing call by some quarters to underplay the role of procedural knowledge in favor of conceptual knowledge (Rittle-Johnson et al. 2001), teachers need to develop a repertoire of both these streams of knowledge as 
these are legitimate and necessary parts of the corpus of knowledge used by learners. In this sense, conceptual and procedural knowledge are important components of preservice and in-service teachers' specialized content knowledge, and the investigation of this knowledge is a major aim of this study.

The examination of conceptual and procedural knowledge in mathematical understanding and subsequent performance also addresses a topical issue for mathematics teachers, the research community, and other stakeholders (Council of the Australian Government 2008) about the link between teaching and the quality learning in mathematics. In relation to this issue, Skemp (1976) took the early steps in highlighting the relative roles of the two in characterizing mathematical performance by focusing on instrumental and relational understanding. Instrumental understanding involves knowing a rule and being able to use it. Instrumental understanding, we argue, is based on and supported by procedural knowledge. Relational understanding is about knowing what to do and why. In order to demonstrate relational understanding, teachers will have to draw on their conceptual knowledge.

More recently, Chinnappan and Chandler (2010) elucidated the role of mathematics teachers' conceptual knowledge in reducing information processing loads that could be associated with problem solving. In a similar vein, the decoding of structures underlying complex mathematics concepts, which is necessary for deep mathematical understanding, was deemed to be buttressed by robust conceptual knowledge, both by teachers and learners (Mason et al. 2009). Taken together, these studies highlight the need to improve current understandings about the conceptual-procedural knowledge interface.

\section{Conceptual framework}

The research questions and the interpretation of data in the present study were guided by a representational reasoning (RR) model of mathematical understanding developed by Barmby et al. (2009). This is a theoretical model of understanding mathematical concepts. In developing the model, Barmby et al. were concerned with both the characterization and demonstration of understanding. Central to the RR model of understanding is the notion of connections or relations between mental representations of a concept. The RR model posits that individuals' depth of understanding is dependent on the quality of their internal representations of a concept and the relationships among these representations. These relationships can be established in varied ways, but the key to building these relationships are reasoning processes. While individual internal representations cannot be directly examined, inferences about understanding can be made from students' demonstrations of external representations and the quality of the connections they make between them. For example, the concept of multiplication of whole numbers can be represented in several ways, including (a) repeated addition, (b) rows and columns in a rectangular array, and (c) operations in a lattice algorithm. All three constitute defensible representations of the concept of multiplication. However, the lattice algorithm can be explained purely from a procedural perspective with little or no reference to the place value idea it embodies. Thus, a representation does not necessarily indicate a holistic understanding of the nuances of a particular mathematics concept. In addition, the complexities inherent in a concept need to be 
demonstrated by robust reasoning that foregrounds the procedural and conceptual aspects of that concept.

The decision to use the RR model was based on the desire to better understand, capture, and support pre-service teachers' abilities to construct a more balanced conceptual and procedural knowledge in the area of fractions. In both phases of the study, we developed a coding system to evaluate the quality of pre-service teachers' conceptual and procedural knowledge. The coding system necessitated an analysis of representations, connections among those representations, and quality of those connections (if any). Barmby et al.'s RR model guided us in this important process. The representational framework also provided a powerful theoretical lens into the depth of understanding of fractions and how to promote that understanding in teaching. Thus, our representational reasoning teaching and learning (RRTL) approach to instruction, developed and implemented in phase 2 of the present study, drew on Barmby et al.'s model.

\section{Purpose and research questions}

The purposes of the study are to (a) examine the quality of pre-service teachers' representations of fraction concepts in terms of their demonstration of procedural and conceptual knowledge and (b) assess the impact of a representational reasoning teaching and learning (RRTL) instructional strategy on the quality of pre-service teachers' procedural and conceptual understanding of fraction concepts and operations. This was addressed by generating data relevant to the following research questions:

What is the relative use of procedural and conceptual knowledge when pre-service teachers represent fraction operations?

Does a representational reasoning teaching and learning approach have an impact on the development of pre-service primary teachers' procedural knowledge of fractions? Does a representational reasoning teaching and learning approach have an impact on the development of pre-service primary teachers' conceptual knowledge of fractions?

\section{Methodology}

Research design and plan

There were two major phases in this study, with each phase utilizing different designs. In phase 1 of the study, a descriptive research design was adopted while phase 2 involved the use of a post-test only non-experimental research design. Phase 2 did not follow a randomized control group, as the study was conducted within an existing teacher education course and there was no access to an equivalent control group. In such designs, it is suggested that the treatment is sustained (Creswell 2003) and detailed so that the researcher is able to make claims about the impact of the intervention which in this case involved the introduction of RRTL to the participants. The above research 
strategy allowed the identification of participants' quality of knowledge in the area fractions (phase 1) which was used to develop and assess a more effective teaching program (phase 2).

Phase 1 involved four key stages: a cohort of pre-service teachers (cohort 1) participated in a 13-week subject involving a 2-h lecture and 1-h tutorial per week (stage 1). At the end of this subject, the pre-service teachers completed a 14-item examination (stage 2). Analysis of their performance provided information about preservice teachers' misconceptions in a number of areas of the primary curriculum including fractions. In stage 3, pre-service teachers' misconceptions in fractions were analyzed which in turn provided directions for the analysis of their procedural and conceptual knowledge (stage 4). Data about pre-service teachers' procedural and conceptual knowledge guided the development of a teaching program (RRTL), the impact of which was assessed with a second cohort of pre-service teachers (cohort 2) in phase 2 of the study.

\section{Participants}

There were two phases to the current study undertaken with two cohorts of participants completing a first year core mathematics content and pedagogy subject in a Bachelor of Primary Education degree. The Bachelor of Primary Education is a 4-year qualification for future classroom teachers in Australia. During the 4-year period, pre-service teachers complete a series of subjects that focus on content and pedagogy in a number of key learning areas including mathematics.

Cohorts 1 and 2 consisted of a very similar mix of participants in terms of gender, maturity, and previous primary and secondary school mathematics studies. To complete the degree, students needed to have successfully completed specified minimum levels of high school mathematics or undertake additional specified mathematics subjects within their degree. These additional subjects were offered in the second year of their degree.

Phase 1 of the study involved 109 participating pre-service teachers (cohort 1), 95 were female $(87.2 \%)$ and 14 were male $(12.8 \%)$. Twenty-three participants $(21.1 \%)$ in this cohort were classified as mature-age students (21 years or older) when they commenced their degrees. Twenty-five $(22.9 \%)$ participants had not completed the minimum required level of mathematics at high school.

Phase 2 involved 114 participating pre-service teachers undertaking the same subject the following year (cohort 2). In this cohort, 101 participants were female $(88.6 \%)$ and 13 were male $(11.4 \%)$. Thirty-four participants $(29.9 \%)$ in this cohort were classified as mature-age students when they commenced their degrees. Thirty-six $(31.6 \%)$ participants had not completed the minimum required level of mathematics at high school.

Participants in both cohorts had completed mandatory mathematics in the first 10 years of schooling. The topic of fractions is covered comprehensively in all stages of the primary and secondary school syllabi (Board of Studies NSW 2002, 2003). All the concepts and algorithms covered in the core mathematics content and pedagogy subject, in which this study was undertaken, had been mandated in the high school mathematics curriculum. Thus, in relation to the participants' knowledge of fractions prior to their university studies, it can reasonably be assumed that participants had been 
taught common fraction concepts, operations, and associated algorithms in their primary and high school mathematics curriculum.

\section{Fraction tasks}

A set of four fraction tasks were utilized in this research. These tasks were drawn from the examination that was completed at the conclusion of the mathematics content and pedagogy subject undertaken by cohort 1 (phase 1) and cohort 2 (phases 2) of this study. This study focuses on two tasks in each of these examination papers (phase 1tasks 1 and 2; phase 2-tasks 3 and 4) which examine the procedural and conceptual knowledge of fraction concepts and fraction operations.

Phase 1 tasks

Phase 1 of this project involved an analysis of cohort 1's responses in two fraction operation tasks in the final examination of the first year core content and pedagogy subject. These particular tasks were chosen to assess participants' conceptual and procedural knowledge of fractions and fraction algorithms. Participants had been able to engage with similar questions throughout the session in order to consolidate their procedural and conceptual understandings.

Task 1 Subtraction problem involving a mixed number and fractions with different denominators

$$
1 \frac{2}{5}-\frac{5}{6}
$$

Pre-service teachers were asked to complete the calculation and provide an explanation for their operation.

There are several separate procedures involved in solving this problem: changing the mixed number to an improper fraction, identifying the lowest common denominator of the minuend and subtrahend, changing the minuend and subtrahend to equivalent fractions, performing the subtraction, and checking if the answer can be simplified.

Conceptual knowledge of this problem involves an understanding that the minuend and subtrahend are related to the same size "whole," $1 \frac{2}{5}$ is the same as $\frac{7}{5}$ because one whole is the same as $\frac{5}{6}$, equivalent fractions are the same size, subtraction of the subtrahend involves removing 25 lots of $\frac{1}{30}$.

Task 2 Multiplication problem involving fractions

$$
\frac{1}{4} \times \frac{2}{3}
$$

Procedurally, the most efficient solution involves multiplying the numerators together, multiplying the denominators together, and simplifying the answer. 
A conceptual understanding of this task involves the notion that $\frac{1}{4} \times \frac{2}{3}$ involves finding $\frac{1}{4}$ of $\frac{2}{3}$. or $\frac{2}{3}$ of $\frac{1}{4}$. It involves partitioning $\frac{2}{3}$ into four equal parts to find $\frac{1}{4} \times \frac{2}{3}$ or cutting $\frac{1}{4}$ into three equal parts to find $\frac{2}{3}$ of $\frac{1}{4}$.

In undertaking the tasks, pre-service teachers were expected to demonstrate all the procedural and conceptual elements involved in the multiplication of fractions and utilize appropriate visual representations to demonstrate their thinking. To complete the calculations, participants needed to use an appropriate algorithm for carrying out a particular operation with fractions. The successful use of an algorithm would indicate that participants have a procedural understanding, what Skemp (1976) defined as instrumental knowledge (knowing a rule and being able to use it). Conceptual or relational understanding is knowing what to do and why (Skemp 1976). In this case, it involves a comprehension of the nature of fractions (equal parts of a whole object or group) including the meaning of the common fraction symbols (as opposed to the misconception common among children that the numerator and denominator are simply two whole numbers) (NSW Department of Education and Training 2003). Additionally, a conceptual understanding of these tasks involves grasping the partitive nature of multiplying by a fraction and the role of making equivalent fractions in enabling the subtraction of fractional parts.

Phase 2 tasks

Task 3 Addition problem involving a mixed number and fractions with different denominators

$$
1 \frac{5}{6}+\frac{2}{3}
$$

As in phase 1, pre-service teachers were asked to complete the calculation and provide an explanation for their operations.

Two common algorithms could be used to complete this task, either involving most or all of these procedures: changing the mixed number to an improper fraction, identifying a common denominator of the addends, changing the addends to equivalent fractions, performing the addition, and checking if the answer can be simplified.

A conceptual knowledge of this task involves these elements as follows: when the addends are represented visually, the wholes to which they relate need to be the same size; equivalent fractions, e.g., $1 \frac{5}{6}$ is the same size as $\frac{11}{6}, \frac{2}{3}$ is the same size as $\frac{4}{6}, \frac{15}{6}$ is the same size as $2 \frac{3}{6}$ which is the same size as $2 \frac{1}{2}$; addition involves joining two or more quantities together.

Task 4 Division problem involving a mixed number and fraction

$$
1 \frac{1}{2} \div \frac{1}{4}
$$


Two algorithms could be used to complete this task. Firstly, pre-service teachers could change the mixed number into an improper fraction, invert the divisor, multiply the numerators and denominators, and check if the answer can be simplified. Alternatively, pre-service teachers could change the mixed number into an improper fraction, identify a common denominator of the dividend and divisor, change the dividend and divisor to equivalent fractions, divide the numerators and denominators, and check if the answer can be simplified.

A conceptual understanding of this task involves the notion that $1 \frac{1}{2} \div \frac{1}{4}$ involves finding how many quarters are in $1 \frac{1}{2}$. Partitioning $1 \frac{1}{2}$ into quarters and counting the number of quarters will achieve an answer of six.

\section{Coding scheme}

Participants' responses to each of the above four tasks were analyzed in terms of the evidence of conceptual and procedural knowledge and coded as per the scheme below (Table 1).

The coding system was designed to capture and analyze participants' demonstration of conceptual and/or procedural knowledge of fractions. Pre-service teachers were expected to provide evidence of these two strands of knowledge by generating different representations of fraction concepts, fraction operations, and the robustness of their reasoning about relations between these representations. These representations included algorithms and illustrations of fraction operations and associated explanations of their thinking. As indicated earlier, Barmby et al. (2009) model of representational reasoning guided the above system of coding. According to this model, the quality of understanding and related knowledge can be captured by identifying representations and connectedness among these representations that are constructed by learners. Thus, there are two key elements to characterizing conceptual and/or procedural knowledge of fractions. Firstly, we had to describe the representation and secondly, we had to make judgment about the quality of relations that are built between and among these representations. The range of codes used by us reflects both the dimensions used by the participating pre-service teachers.

The initial coding scheme did not include code 3 because participants in cohort 1 did not evidence conceptual knowledge without demonstrating procedural knowledge in their solutions. In phase 2 , it was necessary to include an additional code to provide for this new category of responses from cohort 2 to the division task. The examination questions did ask students to provide reasoning or explanation for their use of the algorithms. While a few students voluntarily demonstrated the ability to complete a procedure in more than one way (see Fig. 1 below), the ability to use procedures flexibly was not assessed by the examination tasks. 
Table 1 Coding scheme for analyzing procedural (PK) and conceptual knowledge (CK) and examples for each category

Code 0: No evidence of Procedural Knowledge (PK) or Conceptual Knowledge (CK). No or incorrect algorithm, illustration and/or explanation.

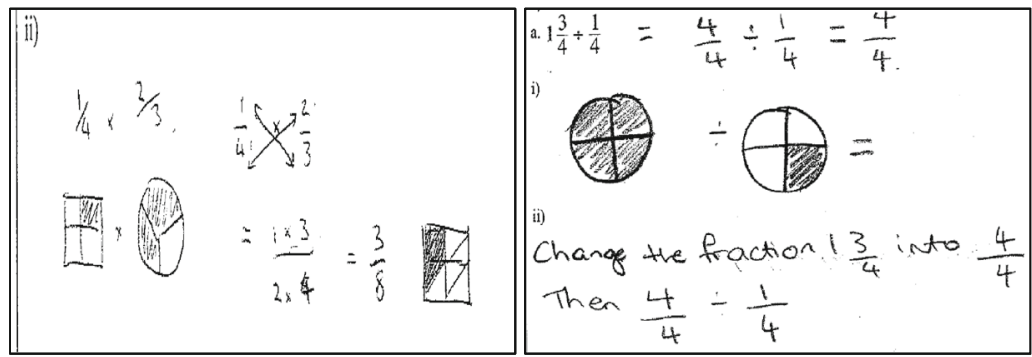

Code 1: PK only. Correct algorithm, with an incorrect or no illustration and/or explanation.

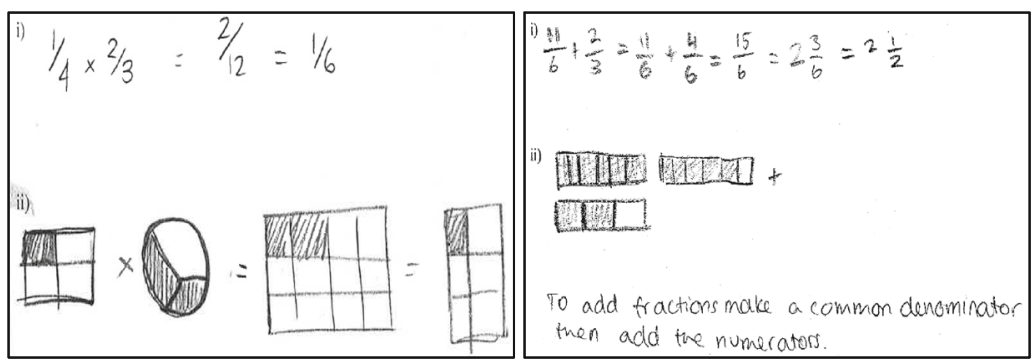

Code 2: PK and some CK. Correct algorithm and while the illustration and/or explanation does not demonstrate an understanding of the operation it does demonstrate a basic conceptual understanding of an important fraction concept rather than just representing the question and answer.

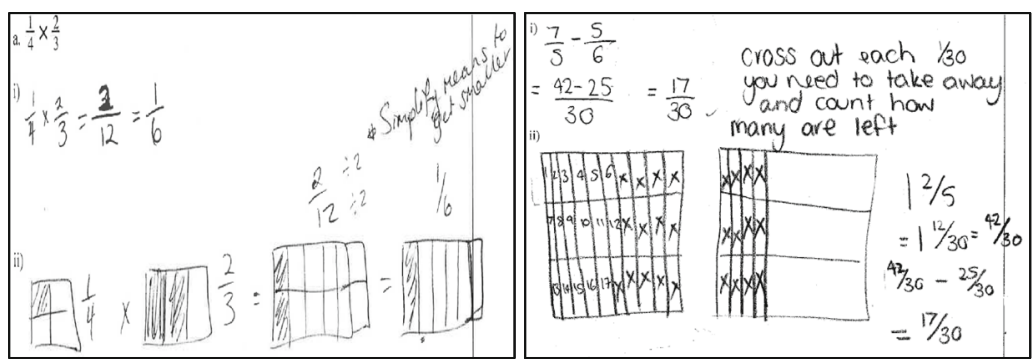

\section{Inter-rater reliability analysis}

In order to determine the reliability of the coding scheme, the extent to which two coders agreed was assessed when they independently categorized participants' responses to the multiplication problem. The two researchers coded ten participants' responses 
Table 1 (continued)

Code 3: CK only. No algorithm or incorrect algorithm. Conceptual illustration and/or explanation which achieved a correct solution. This code was only used in Phase 2 for the division task.
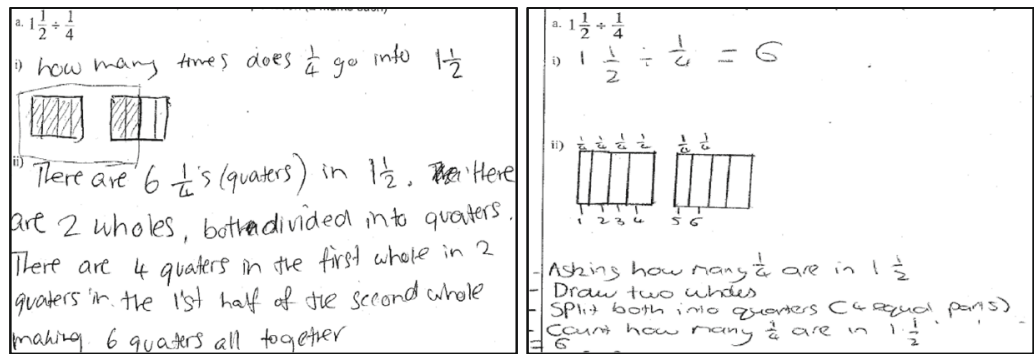

Code 4: PK and CK. Correct algorithm, illustration and/or explanation demonstrating procedural and conceptual knowledge. The illustration and/or explanation demonstrate a conceptual understanding of the operation. Conceptual understanding of every part of the process is not demonstrated.
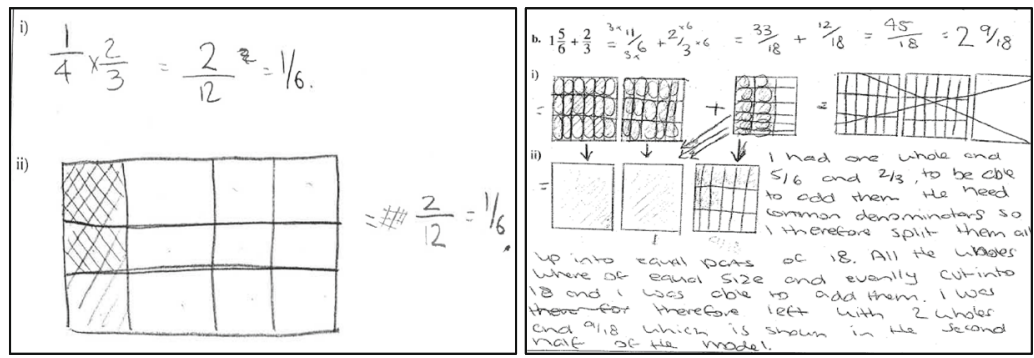

Code 5: $\quad$ PK and strong CK. Algorithm, illustration and/or explanation demonstrate procedural knowledge and strong conceptual knowledge of the fraction concepts and operation.
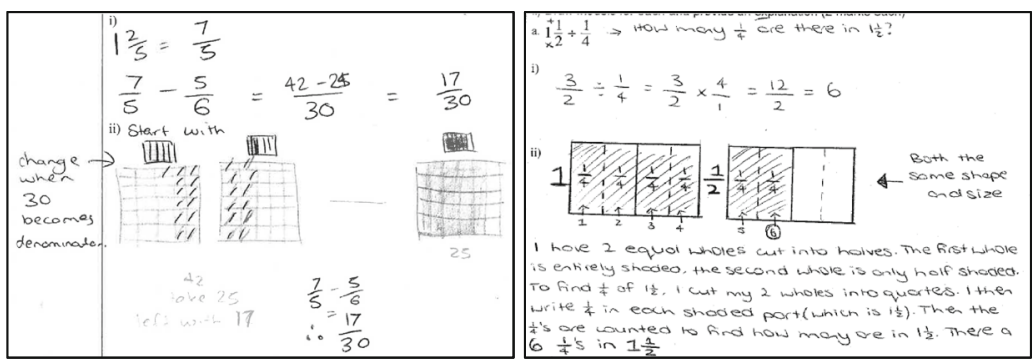

independently. The inter-coder reliability analysis, using the Kappa statistic, was performed to determine consistency. The inter-coder reliability was found to be Kappa $=0.85$ $(p<0.001), 95 \%$ CI $(0.66,1.04)$, indicating substantive agreement (Landis and Koch $1977)$ in the way the participants' responses were coded by each researcher. Potential 


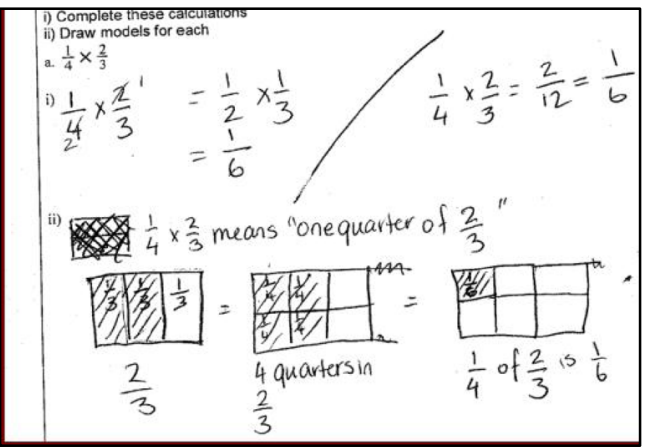

Fig. 1 Demonstration of the ability to complete a procedure in more than one way

areas of disagreement were analyzed which helped us to improve the distance between the codes, thereby reducing areas of ambiguity.

Phase 1: analysis of procedural and conceptual knowledge - subtraction and multiplication problems

\section{Delivery of the content and pedagogy subject to cohort 1}

The delivery of this subject included a weekly 2-h lecture and 1-h tutorial over 13 weeks, in the second session of the degree. Students were provided with a pool of 32 possible examination questions at the beginning of the semester, in their subject outlines, covering number, measurement, patterns, and algebra content. These questions were designed to examine both content and pedagogical knowledge.

One lecture and one tutorial focused exclusively on fraction concepts and operations. Students were given a pool of five examination questions covering these concepts with the understanding that the values would be changed in the examination questions. The fraction lectures and associated tutorials involved explicit teaching of fraction concepts and operations.

Hands-on activities were provided to engage students concretely with important fraction concepts. For example, to support the development of the concept of equivalence, all students engaged in a paper folding activity demonstrating the equivalence of $\frac{1}{3}, \frac{2}{6}, \frac{4}{12}$, and $\frac{8}{24}$ and highlighting the relationship between the activity and the symbolic representations of equivalent fractions.

While a variety of algorithms were supported in a fraction presentation provided on the elearning site, the lecture and tutorial focused on the following common algorithms for fraction operations:

For the addition and subtraction of fractions with different denominators, equivalent fractions were made before adding the fractions and simplifying the answers. e.g.,

$$
\text { Addition : } \frac{1}{2}+\frac{3}{4}=\frac{2}{4}+\frac{3}{4}=\frac{5}{4}=1 \frac{1}{4}
$$




$$
\text { Subtraction : } 1 \frac{1}{4}-\frac{2}{3}=\frac{5}{4}-\frac{2}{3}=\frac{15}{12}-\frac{8}{12}=\frac{7}{12}
$$

For the multiplication, multiplying numerators and multiplying denominators before simplifying the answers, e.g.,

$$
\text { Multiplication : } \frac{1}{2} \times \frac{2}{3}=\frac{2}{6}=\frac{1}{3}
$$

For division, inverting the divisor and multiplying then simplifying, e.g.,

$$
\text { Division : } \frac{1}{2} \div \frac{1}{4}=\frac{1}{2} \times \frac{4}{1}=\frac{4}{2}=2
$$

Lecturer-designed animated PowerPoint slides showing representations of fraction concepts and operations were shown in the lecture to support an understanding of the concepts and procedures and subsequently provided on the elearning site for study access. Opportunities were offered in tutorials and additional support sessions to revise these concepts and procedures. The teaching focus of this subject was to support students to be able to utilize the fraction operation algorithms with conceptual understanding of the operations and associated concepts such as equivalence.

The focus of the study, in terms of fraction instruction, was to ensure these preservice teachers understood basic fraction concepts and could utilize fraction operation algorithms with confidence and understanding of both the procedures and underlying concepts.

The lecture and tutorial explicated the concepts of the following:

The part-whole meaning of fractions including area/regional, measurement, and discrete models.

The quotient meaning of fractions.

The ratio meaning of fractions.

Fractions as operators causing quantities to enlarge or reduce.

Equivalence of fractions.

The language that supports conceptual understanding of operations and concepts.

The considerations involved in connecting fractions as numbers to fractions as parts of things.

The analysis of pre-service teachers' knowledge in phase 1 of the present study is based on a subtraction (task 1) and multiplication problem (task 2), both of which were decontextualized. It was decided that decontextualized problems would provide a high degree of focus on procedural and conceptual development with opportunities to generate a number of representations. Further, our selection of these tasks was guided by the need to provide participants with opportunities to demonstrate multiple layers of procedural and conceptual knowledge and to engage with the mathematics underlying the mathematical concepts involved in operations with fractions in their construction of appropriate representations. 
Phase 2 - the introduction of a representational reasoning teaching and learning (RRTL) approach

\section{Delivery of the content and pedagogy subject to cohort 2}

The performance of cohort 1 in the above-mentioned two examination questions led the authors to analyze responses in order to gain an understanding of the difficulties experienced by these pre-service teachers and address their learning needs. The following concepts were identified as problematic and were considered a priority to address:

The difference between fractions as numbers and fractions as parts of things and the need to represent fractions as equal parts of same sized wholes when representing them in decontextualized examples (Figs. 1 and 3 above).

The notion of equivalence as it relates to mixed numbers, improper fractions, and equivalent fractions for the purpose of adding, subtracting, and simplifying fractions (Fig. 6 above).

An understanding of fractions as operators as they enlarge or reduce quantities (see Figs. 1 and 3 above).

Utilizing Barmby et al.'s (2009) notion that robust mathematical understanding is demonstrated when learners can construct and utilize multiple representations of mathematical ideas and can justify the relationships among representations, our focus shifted from providing representations and activities to support the development of fraction concepts and associated operation algorithms to enabling the pre-service teachers to actively engage in developing and explaining representations of fraction concepts and operations themselves.

In line with the initial delivery of this subject to cohort 1 , the subsequent delivery to cohort 2 included a weekly 2-h lecture and 1-h tutorial over 13 weeks in the second session of the academic year. The pool of possible examination questions in the subject outline increased from 32 to 36 , but the mathematical content covered was identical. The five possible fraction examination questions remained, with an additional question provided which focused on children's common errors in representing fractions (see Fig. 2 below). This particular example question was aimed at challenging some preservice teachers' misconceptions about fractions as numbers and fractions as parts of things that had been problematic for some students in cohort 1. It aimed to engage them as potential teachers in clarifying their thinking as they focused on how they might support children to overcome their difficulties. Remembering that this was only one example of possible student misconceptions, they were directed to research other possible misconceptions in their textbook (Booker et al. 2010) in preparing for their examination.

While animated PowerPoint representations were used in lectures to both cohorts, there was a pedagogical shift from using these representations to scaffold pre-service

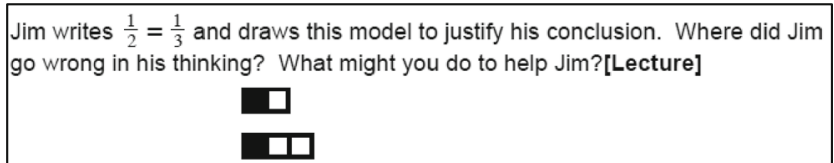

Fig. 2 Exam reflection question focusing on children's misconceptions 
teachers' understanding of fractions and fraction operations to engaging participants in drawing and explaining their own representations. Participants were encouraged to think about "why" algorithms worked rather than just "how" to use them to obtain a correct solution, in terms of fractional concepts and operations.

Lecturers and tutors also undertook explicit teaching involving modeling possible conceptual representations and written explanations of fraction concepts and operations. Important ideas including fractional concepts and misconceptions evident in preservice teachers' representations were discussed concerning the following:

Fractions as numbers and fractions as parts of things (the connections and differences).

Fractions as equal parts of a whole (object or group).

The relationship of parts to the whole (object or group).

The need to use the same size wholes when representing fraction operations.

Equivalence - demonstrated by creating the same size pieces of the same size wholes.

Fractions as operators causing quantities to enlarge or reduce.

The language that might be used to support conceptual understanding of operations and concepts.

Regional models of fraction concepts and operations were the focus of most of the learning activities, although number line representations and discrete models were also discussed and attempted by some pre-service teachers. The focus in the RRTL was the development of a holistic understanding of fractions that was conceptually rich and robust, where pre-service teachers could explain and generate multiple representations of fractional concepts and operations.

\section{Data analysis}

Quantitative data analyses in both phases of the study were conducted with the aid of SPSS version 18. The scale of the data was nominal and ordinal.

The relative roles of procedural and conceptual knowledge used by pre-service teachers as they attempted to represent four fraction operation problems were examined. The general issue of pre-service teachers' proclivity to draw on these knowledge components was examined in terms of the three research questions. The data relevant to each question are presented below.

Research question 1 - what is the relative use of procedural and conceptual knowledge when pre-service teachers represent fraction problems?

\section{Phase 1}

The analysis of phase 1 data includes only five of the six categories of problem representations (Table 1) (code 3 was developed in phase 2 of the study to accommodate a new category of responses that emerged). Figures. 3 and 4 show the results for cohort 1 in an analysis of their use of procedural 


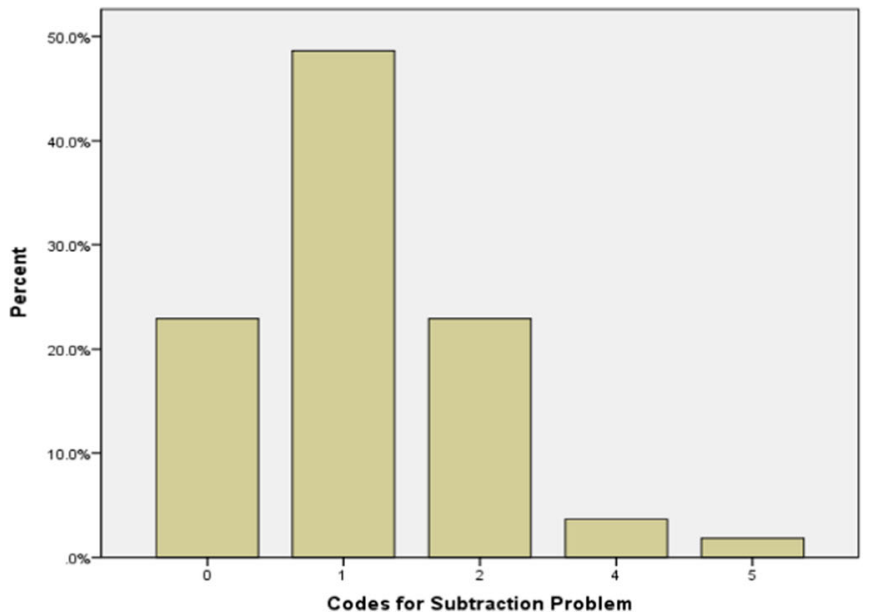

\begin{tabular}{|c|l|}
\hline \multicolumn{2}{|c|}{ Key } \\
\hline Code & Knowledge \\
\hline 0 & None \\
\hline 1 & PK only \\
\hline 2 & PK + minor CK \\
\hline 3 & CK only \\
\hline 4 & PK + good CK \\
\hline 5 & PK + solid CK \\
\hline
\end{tabular}

Fig. 3 Pre-service teachers' responses to subtraction problem: percentages for knowledge categories

knowledge (PK) and conceptual knowledge (CK) when providing solutions to the subtraction and multiplication tasks.

\section{The subtraction problem}

A considerable number of responses $(22.94 \%)$ demonstrated neither procedural nor conceptual knowledge (code 0 ) in the subtraction problem. While a total of $77.06 \%$ of participants in cohort 1 demonstrated procedural knowledge, almost half of the cohort (48.62\%) activated only procedural knowledge in completing the subtraction problem (code 1). In cohort 1, $22.94 \%$ demonstrated procedural knowledge and the group could also demonstrate conceptual knowledge of an important fraction concept (code 2). Only $5.5 \%$ of participants elucidated both conceptual knowledge and procedural knowledge of the operation (codes 4 and 5), and there were no instances of this cohort demonstrating any conceptual knowledge (code 3 ) in coming to a solution. Thus, overall, while a large

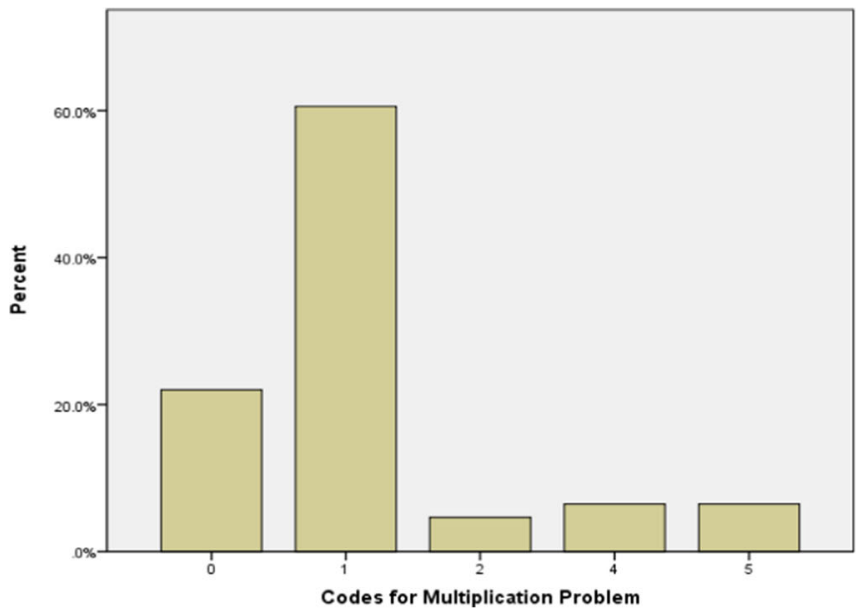

\begin{tabular}{|c|l|}
\hline \multicolumn{2}{|c|}{ Key } \\
\hline Code & Knowledge \\
\hline 0 & None \\
\hline 1 & PK only \\
\hline 2 & PK + minor CK \\
\hline 3 & CK only \\
\hline 4 & PK + good CK \\
\hline 5 & PK + solid CK \\
\hline
\end{tabular}

Fig. 4 Pre-service teachers' responses to multiplication problem: percentages for knowledge categories 
proportion of participants could utilize procedural knowledge only, a small proportion of the cohort could demonstrate a conceptual knowledge of the subtraction concepts and operation.

\section{The multiplication problem}

About one-fifth of responses (22.02\%-code 0$)$ evidenced neither procedural nor conceptual knowledge (code 0 ). A total of $79.69 \%$ of cohort 1 participants demonstrated procedural knowledge. In the cohort, $60.55 \%$ (code 1) activated procedural knowledge only in the multiplication problem (code 1). A further $4.59 \%$ demonstrated procedural knowledge and could also demonstrate conceptual knowledge of an important fraction concept (code 2). Of the participants, $12.84 \%$ demonstrated both conceptual and procedural knowledge of the operation (codes 4 and 5) (Fig. 4). Again, the predominance of procedural in comparison to conceptual knowledge in the multiplication problem is noted.

A two-way contingency table analysis was conducted to evaluate whether representation categories in the subtraction problem were associated with those for the multiplication problem (Table 2). The two variables were the subtraction problem with five levels of representation and the multiplication problem with five levels of representation.

Spearman rank-order correlations were conducted in order to determine if there were any relationships between the pre-service teachers' responses to subtraction and multiplication problems. A two-tailed test of significance indicated that there was a significant positive relationship between the codes for subtraction and multiplication problem, $r s(109)=0.50, p<0.000$. That is, participants, as a group, tended to activate similar levels of procedural or conceptual knowledge along the five codes for both the problems.

\section{Phase 2}

Figures 5 and 6 show the results for cohort 2 in an analysis of their use of procedural and conceptual knowledge when providing solutions to the addition and division tasks.

\section{The addition problem}

In cohort 2, $47.37 \%$ of participants activated both procedural and considerable conceptual knowledge in completing the addition problem (code 4 and 5) with an

Table 2 Codes for multiplication problem vs. codes for subtraction problem cross tabulation

\begin{tabular}{llllllll}
\hline & & \multicolumn{3}{l}{ Codes for subtraction problem } & & & Total \\
\cline { 3 - 6 } & & 0 & 1 & 2 & 4 & 5 & \\
\hline Codes for multiplication problem & 0 & 11 & 12 & 1 & 0 & 0 & 24 \\
& 1 & 13 & 37 & 14 & 0 & 2 & 66 \\
& 2 & 0 & 3 & 2 & 0 & 0 & 5 \\
& 4 & 1 & 1 & 3 & 2 & 0 & 7 \\
Total & 5 & 0 & 0 & 5 & 2 & 0 & 7 \\
\hline
\end{tabular}




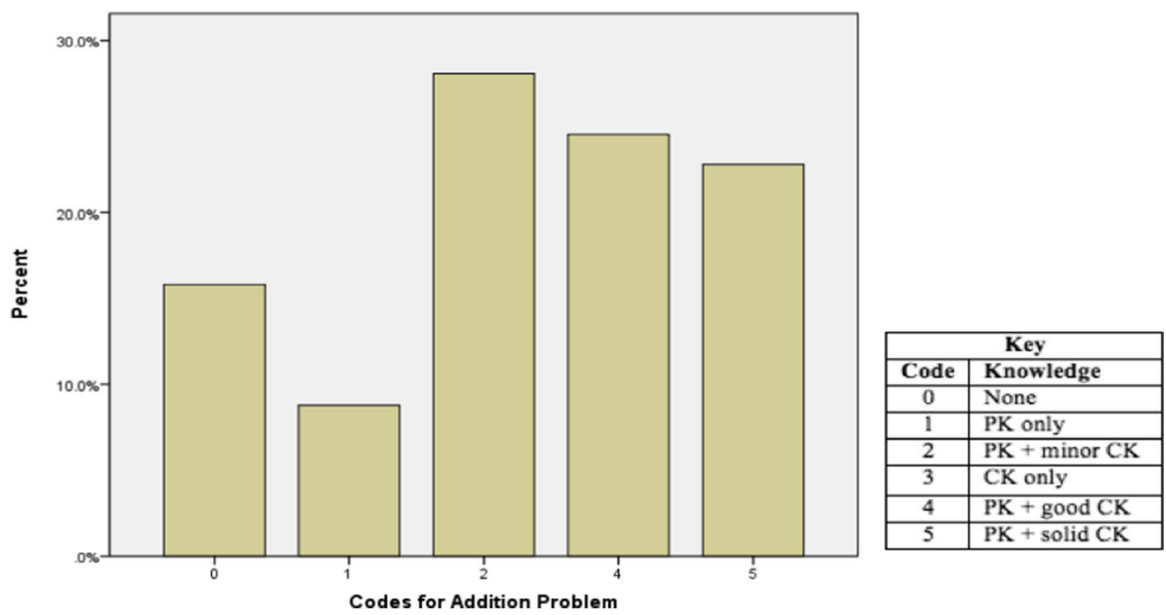

Fig. 5 Pre-service teachers' responses to addition problem: percentages for knowledge categories

additional $28.07 \%$ showing procedural and some conceptual understanding of an important fraction concept (code 2) (Fig. 5). A further $8.77 \%$ elucidated only procedural knowledge (code 1), with $15.79 \%$ of participants evidencing neither procedural nor conceptual knowledge (code 0 ).

\section{The division problem}

In the division problem, nearly $65 \%$ of participants in cohort 2 activated both procedural and conceptual knowledge (codes 4 and 5), in comparison to the $13.16 \%$ of participants demonstrating procedural knowledge only (code 1) (Fig. 6). An additional group of responses emerged from this problem (code 3 ) with nearly $8.77 \%$ of the cohort utilizing conceptual knowledge to arrive at a correct solution without using an algorithm. About $12.28 \%$ evidenced neither procedural nor conceptual knowledge (code 0$)$.

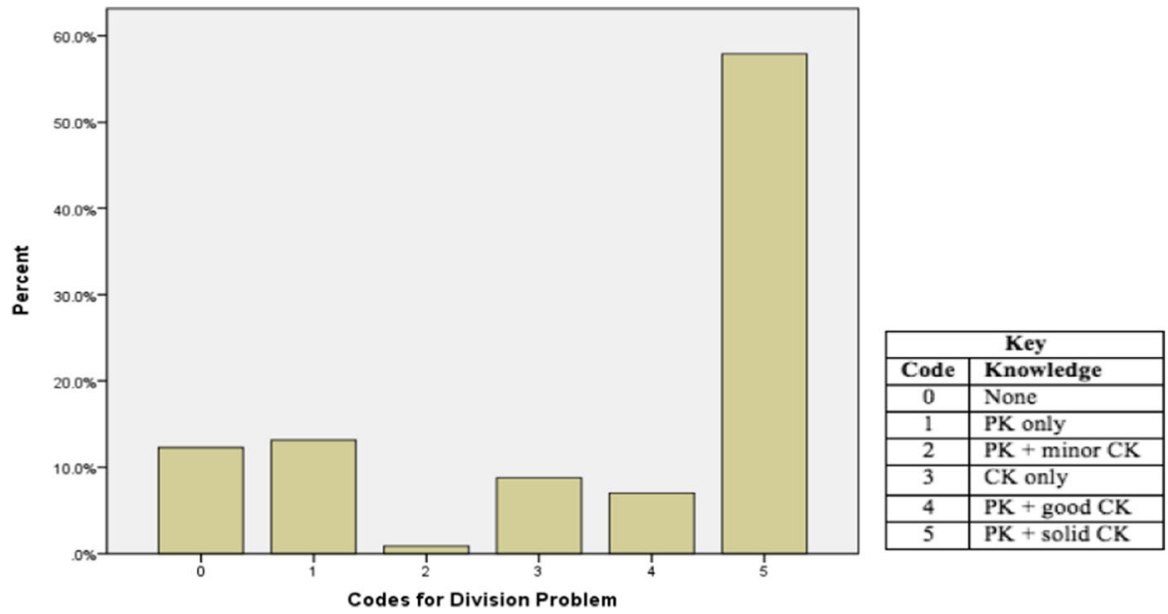

Fig. 6 Pre-service teachers' responses to division problem: percentages for knowledge categories 
Again, a two-way contingency table analysis was conducted to evaluate whether representation categories in the addition problem were associated with those for the division problem (Table 3).

As before, Spearman rank-order correlation analysis indicated that the quality of representations in both the addition and division problems was found to be significantly related, $r s(109)=0.50, p<0.000$.

Research question 2-does a representational reasoning teaching and learning approach have an impact on the development of pre-service primary teachers' procedural knowledge of fractions?

\section{A comparison of phase 1 and phase 2 results}

Due to the changes in the operations examined with cohorts 1 and 2, from multiplication to division and subtraction to addition, there are limitations in making direct comparisons. However, the addition and subtraction algorithms are closely related, with the common approach involving converting the fractions to equivalent fractions with the same denominator before adding or subtracting. The common division algorithm involves inverting the divisor and using the multiplication algorithm (it could be reasonably argued that the division algorithm is more complicated and hence more difficult to utilize). While cohorts 1 and 2 are different, their commencing knowledge about fraction operations was assumed to be similar on the basis of their school mathematics experiences.

Having taken this into consideration, it is worth noting that the proportion of participants who were able to find correct solutions to the fraction operation tasks using algorithms did improve within the solution attempts of the two cohorts (Table 4). Comparing the responses of the participants in cohorts 1 and 2 in the subtraction and addition problems, $77.06 \%$ (-) of cohort 1 participants, compared to $84.21 \%(+)$ of the participants in cohort 2 , were able to achieve a correct solution using an appropriate algorithm. A smaller difference was noted in comparing the two cohorts' responses to the multiplication and division tasks with $77.98 \%(\times)$ of participants in cohort 1 and $78.95 \%(\div)$ in cohort 2 demonstrating the competent use of appropriate algorithms.

Table 3 Codes for division problem vs. codes for addition problem cross tabulation

\begin{tabular}{llllllll}
\hline & & \multicolumn{2}{l}{ Codes for addition problem } & & \multirow{2}{*}{ Total } \\
\cline { 3 - 6 } & & 0 & 1 & 2 & 4 & 5 & \\
\hline \multirow{2}{*}{ Codes for division problem } & 0 & 6 & 4 & 0 & 2 & 2 & 14 \\
& 1 & 3 & 4 & 6 & 1 & 1 & 15 \\
& 2 & 0 & 1 & 0 & 0 & 0 & 1 \\
& 3 & 4 & 0 & 0 & 4 & 2 & 10 \\
& 4 & 1 & 0 & 2 & 3 & 2 & 8 \\
Total & 5 & 4 & 1 & 24 & 18 & 19 & 66 \\
& & 18 & 10 & 32 & 28 & 26 & 114 \\
\hline
\end{tabular}


Table 4 Comparison of the utilization of procedural and conceptual knowledge by cohorts 1 and 2

\begin{tabular}{|c|c|c|c|}
\hline \multicolumn{2}{|c|}{ Knowledge type: } & Percentage of cohort $1(\%)$ & Percentage of cohort $2(\%)$ \\
\hline \multicolumn{4}{|c|}{ Procedural (PK) } \\
\hline \multicolumn{4}{|c|}{ Conceptual (CK) } \\
\hline Code & Description & Subtraction & Addition \\
\hline 0 & None & 22.94 & 15.79 \\
\hline 1 & PK only & 48.62 & 8.77 \\
\hline 2 & $\mathrm{PK}+$ minor $\mathrm{CK}$ & 22.94 & 28.07 \\
\hline 4 & $\mathrm{PK}+\operatorname{good} \mathrm{CK}$ & 3.67 & 24.56 \\
\hline 5 & $\mathrm{PK}+$ strong $\mathrm{CK}$ & 1.83 & 22.81 \\
\hline 3 & C only & 0 & 0 \\
\hline \multicolumn{2}{|c|}{ Total of PK } & 77.06 & 84.21 \\
\hline \multicolumn{2}{|c|}{ Total of all CK } & 28.4 & 75.44 \\
\hline \multicolumn{2}{|c|}{ Total of notable CK } & 5.5 & 47.37 \\
\hline \multicolumn{2}{|c|}{ Code } & Multiplication & Division \\
\hline 0 & None & 22.02 & 12.28 \\
\hline 1 & PK only & 60.55 & 13.16 \\
\hline 2 & $\mathrm{PK}+$ minor $\mathrm{CK}$ & 4.59 & 0.88 \\
\hline 4 & $\mathrm{PK}+\operatorname{good} \mathrm{CK}$ & 6.42 & 7.02 \\
\hline 5 & $\mathrm{PK}+$ strong $\mathrm{CK}$ & 6.42 & 57.89 \\
\hline 3 & CK only & 0 & 8.77 \\
\hline \multicolumn{2}{|c|}{ Total of PK } & 77.98 & 78.95 \\
\hline \multicolumn{2}{|c|}{ Total of all CK } & 17.63 & 74.56 \\
\hline \multicolumn{2}{|c|}{ Total of notable CK } & 13.04 & 73.68 \\
\hline
\end{tabular}

Research question 3-does a representational reasoning teaching and learning approach have an impact on the development of pre-service primary teachers' conceptual knowledge of fractions?

\section{A comparison of phase 1 and phase 2 results}

In comparing the conceptual knowledge evidenced in phases 1 and 2 of the study, the improvement was considerable following the introduction of the RRTL approach (Table 4). In attempting the subtraction and addition problems, the majority of responses of cohort 2 were indicative of higher levels of conceptual knowledge activation than those of Cohort 1 , with $75.44 \%$ of cohort $2(+)$ evidencing conceptual knowledge compared to $28.4 \%$ of cohort $1(-)$. Additionally, a significant number of participants in cohort $2(47.37 \%)$ compared to only $5.5 \%$ in cohort 1 showed a strong conceptual understanding of the essential fraction ideas and concepts involved in adding or subtracting fractions.

Similarly, the majority of cohort 2 (74.56\% compared to $17.63 \%$ of cohort 1$)$ exhibited some conceptual understanding of fraction ideas, with nearly all of these 
participants $(73.68 \%)$ being able to successfully represent and explain the important fractional concepts involved (compared to only $13.04 \%$ of cohort 1 ).

The proportion of pre-service teachers who were unable to achieve a correct answer using either procedural and/or conceptual knowledge decreased considerably over the 2 years. Whereas $22.94 \%(-)$ and $22.02 \%(\times)$ of cohort 1 did not achieve correct solutions in the subtraction and multiplication tasks (code 0 ), within cohort 2, $15.79 \%$ $(+)$ and $12.28 \%(\div)$ of participants did not achieve correct solutions within the addition and division tasks (code 0 ).

Interestingly, nearly $8.77 \%$ of cohort 2 were able to achieve a correct answer to the division task $(\div)$ utilizing a representation without using an algorithm. Given that pre-service teachers were required to provide a calculation and explanation, any omission in providing evidence of procedural knowledge can be interpreted as not having this knowledge.

In comparing the results of both cohorts, it seems that a RRTL approach has impacted positively on cohort 2's acquisition and subsequent use of conceptual understanding of fraction operations over the period of this research.

\section{Discussion and implications}

The aims of this study were (a) to describe the state of knowledge of fractions in terms of procedural-conceptual continuum and (b) to track the development of these two strands of knowledge of two cohorts of pre-service teachers, in the context of fraction operation problems, before and after the implementation of a representational reasoning teaching and learning (RRTL) approach. The study was grounded on the assumption that future teachers need to develop a strong body specialized content knowledge of mathematics (Ball, et al. 2005), and this knowledge can be analyzed from a procedural and conceptual perspective. While conceptual knowledge may subsume procedural knowledge and indeed contribute to better procedural knowledge, it is important to capture and support the growth of both strands of knowledge for future teachers of mathematics and numeracy.

In our attempt to generate data about the quality of participants' understanding of fraction operations as revealed by their problem-solving attempts and the impact of an instructional approach on their subsequent understanding, we were guided by the theoretical model of understanding that was developed by Barmby et al. (2009). The model was found to be appropriate for both the construction and use of our coding system and in guiding the instruction for the pre-service teachers.

Research question 1 framed the first phase of the study, the aim being to describe levels of procedural and conceptual knowledge of fractions that were activated in the context of solving subtraction and multiplication problems. Analysis of the quality of representations produced by the participants in cohort 1 indicates that few participants had developed a robust body of conceptual knowledge of subtraction and multiplication of fractions. Further, data suggested that the participants' knowledge was primarily procedural in nature. This was even more pronounced in their responses to the multiplication than subtraction problem. A possible explanation for this may be that multiplying with fractions is somewhat more demanding conceptually than with subtraction. While subtracting fractions has similarities to subtraction involving whole numbers, multiplying by fractions is partitive in nature and results in a product that is 
smaller than the multiplicand (Mack 2001). The high number of errors that were committed in both the subtraction and multiplication tasks suggests that these preservice teachers relied heavily on their procedural knowledge which, when not supported by conceptual understanding, could provide fewer opportunities to review for possible mistakes.

The cross-tabulation analyses of both cohorts' patterns of activating procedural or conceptual knowledge in their responses to both their examination questions were not independent. That is, regardless of the problem type, the content knowledge of cohort 1 was mainly procedural in nature and the content knowledge of cohort 2 was both procedural and conceptual. It is therefore contended by the authors that while both strands of knowledge are necessary for teaching, the predominance of procedural over conceptual knowledge, evidenced in the responses of cohort 1, does not constitute a healthy state. Teachers who develop content knowledge that is predominantly procedural cannot be expected to help children develop rich conceptual connections (Lesh and Zawojewski 2007) and may, in fact, contribute to students' misconceptions (Ma 1999). In analyzing the quality of cohort 1 's content knowledge in fractions and fraction operations, we sought to inform our own future teacher education programs to better support pre-service teachers undertaking our courses. The intent being to develop a more balanced the knowledge they will need to be effective teachers of fraction concepts.

Research questions 2 and 3 were concerned with the impact of a teaching and learning approach (RRTL) based on the development of appropriate representations, including effective algorithms, conceptual illustrations of fraction operations, and written explanations on the development of pre-service teachers' procedural and conceptual knowledge of fractions. The results suggest that while there was a negligible to small positive effect on the development of procedural knowledge, the RRTL approach to teaching and learning had considerable positive impact on pre-service teachers' conceptual knowledge.

The RRTL approach was based on the assumption that pre-service teachers who had developed a robust conceptual knowledge base could be expected to exhibit a commensurate improvement in procedural knowledge. This appears to be the case with most of the participants. However, there were a number of pre-service teachers who demonstrated conceptual knowledge but could not use an appropriate procedure to find a solution. Such instances raise a question about the character of conceptual knowledge in subsuming and supporting procedural knowledge or, indeed, how one influences the other. This issue warrants further investigation.

Pre-service teachers' representations of fraction division problems, particularly from a conceptual point of view, can inform us about their ability to discriminate between measurement versus partitive interpretations, which have been shown to be a problematic area for both experienced and pre-service teachers (Flores 2002; Siebert 2002). In their solution attempts of the division problem, participants used regional models that involved the determination of the number of the second fraction $\left(\frac{1}{4}\right)$ that goes into the first ( $\left.1 \frac{1}{2}\right)$ (measurement interpretation).

The results in the present study also showed that cohort 2 developed a considerably richer conceptual understanding of fraction concepts and operations than cohort 1. 
While we are encouraged by the results, our data are based on a number of decontextualized fraction problems. An investigation of the construction, durability, flexibility, and transfer of conceptual knowledge within contextualized fraction problems would be a worthwhile extension in determining the effectiveness of the RRTL approach. Our future research program aims to examine this question. Equally, an assessment of the value of the RRTL approach, as it applies to other mathematics content areas, can also be expected to inform the impact of the composition and general features of this model of instruction.

Cohort 1's limited conceptual knowledge and dependence on procedural knowledge, evidenced in the results of the examination in phase 1 of this study, provided the impetus for the second phase. Initially, procedural and conceptual knowledge were examined in the context of subtraction and multiplication examination tasks. Subsequently, cohort 2's conceptual and procedural knowledge were examined in addition and division tasks. While this could be seen as a limitation of the study due to the changes in the fraction operations involved, it is our contention that in both groups, there are structural similarities based on the inverse relationships between addition and subtraction as well as multiplication and division, both algorithmically and conceptually. Further, while the operations utilized in both phases were different, the focus of the study was on capturing the state of procedural and conceptual knowledge of fraction concepts and operations in order to better support pre-service teachers in their development.

In making claims about the quality of pre-service teachers' procedural and conceptual knowledge, a coding system was developed that was sensitive, in the main, to the positive aspects of pre-service teachers' responses to the given set of tasks. The focus of the research was to identify the evidence of conceptual and procedural knowledge rather than to analyze the nature of conceptual and/or procedural misunderstandings. Future studies could extend the coding to explicitly analyze the types of errors committed by participants.

The claims made about the quality of knowledge demonstrated by participants in this study are based on participants being given explicit instructions to complete the calculations, draw a representation of the concepts and operations, and explain their representations. Within the context of a quantitative study involving a substantial number of participants, we are comfortable in making the above claim. However, it would be worthwhile interviewing participants individually with a view to gaining further insights into the quality of their conceptual and procedural knowledge of fractional concepts and operations. Such detailed data could also provide more information about the factors contributing to the development of their knowledge.

The study was designed to identify changes in pre-service teachers' knowledge of fractions and fraction operations in terms of procedural and conceptual dimensions. To ensure that the nature of the tasks used in the study did not act as a confounding factor, they had several features in common: they were decontextualized, solutions could be found by using algorithms, and concepts could be demonstrated utilizing visual representations and supported by written explanations. It might be argued that there is sufficient variability within these boundaries that could contribute to the changes in knowledge demonstrated by the participants. Such a hypothesis is reasonable and could be investigated through future research by adopting a quasi-experimental design. 
Finally, it is contended that this study contributes significantly in amplifying the nature of specialized content knowledge that has been argued to be important for teachers of mathematics. In drawing attention to this cluster of knowledge, Ball et al. (2008) commented that the "mathematical demands of teaching are substantial" and that there is a need to conceptualize and develop sensitive measures of this knowledge. We have undertaken this task within the context of teachers' knowledge for teaching fractions by drawing on the framework of procedural-conceptual understandings. Specialized content knowledge (Ball et al. 2005) underpins both pre-service and inservice teachers' abilities to identify and understand errors committed by their students and provide an appropriate instruction to improve their students' understanding. Casting specialized content knowledge in terms of procedural-conceptual dimensions thus provides both teachers and researchers with tools to anticipate, analyze, and explain student errors.

\section{Conclusion}

The present study was motivated by a desire to better understand the quality of content knowledge that pre-service teachers develop in a mathematics content and pedagogy course and examine the impact of an instructional model (RRTL) designed to improve their conceptual understanding of fraction concepts and operations. Our results suggest that pre-service teachers come into teacher education programs with knowledge of fractions and fraction operations that is mainly about procedures with limited appreciation of the conceptual basis of these concepts and operations. The results of this study provide support for the argument that procedurally driven fraction knowledge has limited value and, indeed, could impede the development of the specialized content knowledge and pedagogical content knowledge necessary for quality mathematics teaching.

In considering the RRTL approach to pre-service teacher education in the area of fractions, the results reported here are encouraging. Pre-service teachers can be supported, within an existing teacher education program, to construct conceptually and procedurally robust content knowledge through the development of appropriate representations of fraction concepts and operations. Further, our experience showed that the use of representationally rich instruction could enhance the building of the fraction procedural-conceptual knowledge nexus. The authors contend that the impact of RRTL instruction can be further strengthened with focus on pre-service teachers cooperatively generating their own representations and investigating the links between the underlying mathematics and effective algorithms.

Acknowledgments Portions of the work reported here were presented at the Mathematics Education Research Group of Australasia conference, Alice Springs.

\section{References}

Ball, D. L. (1990). The mathematical understanding that preservice teachers bring to teacher education. Elementary School Journal, 90, 449-466. 
Ball, D. L., Hill, H. C., \& Bass, H. (2005). Knowing mathematics for teaching: who knows mathematics well enough to teach third grade, and how can we decide? American Educator, 5(3), 14-17. 20-22, 43-46.

Ball, D. L., Thames, M. H., \& Phelps, G. (2008). Content knowledge for teaching: what makes it special? Journal of Teacher Education, 59(5), 389-407.

Barmby, P., Harries, T., Higgins, S., \& Suggate, J. (2009). The array representation and primary children's understanding and reasoning in multiplication. Educational Studies in Mathematics, $70,217-241$.

Baroody, A. J., Feil, Y., \& Johnson, A. R. (2005). An alternative reconceputalization of procedural and conceptual knowledge. Journal for Research in Mathematics Education, 38(2), 115-131.

Board of Studies NSW. (2002). Mathematics K-6: syllabus 2002. Sydney: Author.

Board of Studies NSW. (2003). Mathematics years 7-10 syllabus. Sydney: Author.

Bobis, J., Higgins, J., Cavanagh, M., \& Roche, A. (2012). Professional knowledge of practicing teachers of mathematics. In B. Perry, T. Lowrie, T. Logan, A. MacDonald, \& J. Greenlees (Eds.), Mathematics education research group of Australasia: research in mathematics education in Australasia 2008-2011 (pp. 313-341). The Netherlands: Sense Publishers.

Booker, G., Bond, D., Sparrow, L., \& Swan, P. (2010). Teaching primary mathematics (4th ed.). Pearson: French's Forest.

Chick, H. (2010). Aspects of teachers' knowledge for helping students learn about ratio. In L. Sparrow, B. Kissane, \& C. Hurst (Eds.), Shaping the future of mathematics education: proceedings of the 33rd annual conference of the mathematics education research group of Australasia (Vol. 1, pp. 145-152). Fremantle: MERGA.

Chinnappan, M., \& Chandler, P. (2010). Managing cognitive load in the mathematics classroom. The Australian Mathematics Teacher, 66(1), 5-11.

Council of the Australian Government. (2008). National numeracy review report. Canberra: Author.

Creswell, J. W. (2003). Research design: qualitative, quantitative and mixed methods approaches. London: Sage publications.

Flores, A. (2002). Profound understanding of division of fractions. In B. Litwiller \& G. Bright (Eds.), NCTM handbook: Making sense of fractions, ratios and proportions (pp. 237-246). Reston: National Council of Teachers of Mathematics.

Frid, S., Goos, M., \& Sparrow, L. (2009). What knowledge is needed for effective teaching of mathematics? Mathematics Teacher Education and Development, 9, 1-3.

Groth, R. E., \& Bergner, J. A. (2006). Preservice elementary teachers' conceptual and procedural knowledge of mean, median, and mode. Mathematical Thinking and Learning, 8(1), 37-63.

Hallett, D., Nunes, T., \& Bryant, P. (2010). Individual differences in conceptual and procedural knowledge when learning fractions. Journal of Educational Psychology, 102(2), 395-406.

Harvey, R. (2012). Stretching student teachers' understanding of fractions. Mathematics Education Research Journal, 24, 493-511.

Hill, H. C., Ball, D., \& Schilling, S. (2008). Unpacking pedagogical content knowledge: conceptualizing and measuring teachers' topic-specific knowledge of students. Journal for Research in Mathematics Education, 39(4), 372-400.

Lamon, S. J. (2007). Rational numbers and proportional reasoning. In F. K. Lester (Ed.), Second handbook of research on mathematics teaching and learning (pp. 629-667). Charlotte: Information Age.

Landis, J. R., \& Koch, G. G. (1977). The measurement of observer agreement for categorical data. Biometrics, $33,159-174$.

Lesh, R., \& Zawojewski, J. (2007). Problem solving and modeling. In F. K. Lester (Ed.), Second handbook of research on mathematics teaching and learning (pp. 763-807). Charlotte: Information Age.

Ma, L. (1999). Knowing and teaching elementary mathematics: teachers' understanding of fundamental mathematics in China and the United States. Mahwah: Lawrence Erlbaum.

Mack, N. K. (2001). Building on informal knowledge through instruction in a complex content domain: partitioning, units, and understanding multiplication of fractions. Journal for Research in Mathematics Education, 32(3), 267-295.

Mason, J., Stephens, M., \& Watson, A. (2009). Appreciating mathematical structure for all. Mathematics Education Research Journal, 21(2), 10-32.

Newton, K. J. (2008). An extensive analysis of pre-service elementary teachers' knowledge of fractions. American Education Research Journal. doi:10.3102/0002831208320851.

NSW Department of Education and Training. (2003). Fractions, pikelets and lamingtons. Ryde: Author.

Rittle-Johnson, B., \& Alibali, M. W. (1999). Conceptual and procedural knowledge of mathematics: does one lead to the other? Journal of Educational Psychology, 91(1), 175-189. 
Rittle-Johnson, B., Siegler, R. S., \& Alibali, M. W. (2001). Developing conceptual understanding and procedural skill in mathematics: an iterative process. Journal of Educational Psychology, 93(2), 346-362.

Schneider, M., \& Stern, E. (2010). The developmental relations between conceptual and procedural knowledge: a multimethod approach. Developmental Psychology, 46(1), 178-192.

Senk, S., Tatto, M. T., Reckase, M., Rowley, G., Peck, R., \& Bankov, K. (2012). Knowledge of future primary teachers for teaching mathematics: an international comparative study. ZDM-The International Journal on Mathematics Education, 44(3), 307-324.

Shulman, L. S. (1986). Those who understand: knowledge growth in teaching. Educational Researcher, 4(15), 4-14.

Siebert, D. (2002). Connecting informal thinking and algorithms: the case of division of fractions. In B. Litwiller \& G. Bright (Eds.), NCTM yearbook: Making sense of fractions, ratios and proportions (pp. 247-256). Reston: National Council of Teachers of Mathematics.

Skemp, R. R. (1976). Relational understanding and instrumental understanding. Mathematics Teaching, 77, 20-26.

Tirosh, D. (2000). Enhancing prospective teachers' knowledge of children's conceptions: the case of division of fractions. Journal for Research in Mathematics, 31(1), 5-25.

Walshaw, M. (2012). Teacher knowledge as fundamental to effective teaching practice. Journal of Mathematics Teacher Education, 15(3), 181-185. 